\title{
FIXED-CIRCLE PROBLEM ON $S$-METRIC SPACES WITH A GEOMETRIC VIEWPOINT
}

\author{
Nihal Y. Özgür and Nihal Taş
}

\begin{abstract}
Recently, a new geometric approach called the fixed-circle problem has been introduced to fixed-point theory. The problem has been studied using different techniques on metric spaces. In this paper, we consider the fixed-circle problem on $S$-metric spaces. We investigate existence and uniqueness conditions for fixed circles of self-mappings on an $S$-metric space. Some examples of self-mappings having fixed circles are also given.
\end{abstract}

Keywords: fixed-circle problem; self-mapping; $S$-metric space.

\section{Introduction}

The existence and uniqueness theorems of fixed points of self-mappings satisfying some contractive conditions have been extensively studied since the time of Stefan Banach (see $[1,2])$. Many authors have investigated new fixed-point theorems on metric spaces or generalizations of metric spaces. For example, Sedghi, Shobe and Aliouche obtained Banach's contraction principle on $S$-metric spaces [12]. We studied some generalizations of Banach's contraction principle on an $S$-metric space [8] and investigated new fixed-point theorems for the following contractive condition (which is called Rhoades' condition [11]) (see [6, 14]):

$$
\begin{aligned}
(S 25) \mathcal{S}(T x, T x, T y)< & \max \{\mathcal{S}(x, x, y), \mathcal{S}(T x, T x, x), \mathcal{S}(T y, T y, y) \\
& \mathcal{S}(T y, T y, x), \mathcal{S}(T x, T x, y)\}
\end{aligned}
$$

for each $x, y \in X, x \neq y$. We then gave the concept of diameter and obtained a new contractive condition using this notion as follows [6]:

$$
(S 25 a) \mathcal{S}(T x, T x, T y)<\operatorname{diam}\left\{U_{x} \cup U_{y}\right\},
$$

for each $x, y \in X(x \neq y)$, where $U_{x}=\left\{T^{n} x: n \in \mathbb{N}\right\}, U_{y}=\left\{T^{n} y: n \in \mathbb{N}\right\}$, $\operatorname{diam}\left\{U_{x}\right\}<\infty$ and $\operatorname{diam}\left\{U_{y}\right\}<\infty$.

Received October 11, 2018; accepted January 18, 2019

2010 Mathematics Subject Classification. Primary 47H10; Secondary 54H25, 55M20, 37E10 
Although the existence of fixed points of functions has been studied on various metric spaces, there is no study on the existence of fixed circles. Therefore, the fixed-circle problem arises naturally. There are some examples of functions with a fixed circle on some special metric spaces. For example, let $\mathbb{C}$ be an $S$-metric space with the $S$-metric

$$
\mathcal{S}(z, w, t)=\frac{|z-t|+|w-t|}{2},
$$

for all $z, w, t \in \mathbb{C}$. Let the mapping $T$ be defined as

$$
T z=\frac{1}{\bar{z}},
$$

for all $z \in \mathbb{C} \backslash\{0\}$. The mapping $T$ fixes the unit circle $C_{0,1}^{S}=\{x \in X: \mathcal{S}(x, x, 0)=1\}$.

Recently, Özdemir, İskender and Özgür used new types of activation functions having a fixed circle for a complex valued neural network [5]. The usage of these types activation functions leads us to guarantee the existence of fixed points of the complex valued Hopfield neural network (see [5] for more details).

Hence it is important to investigate some fixed-circle theorems on various metric spaces. In [9], we obtained some fixed-circle theorems on metric spaces. We studied some existence theorems for fixed circles with a geometric interpretation and gave necessary conditions for the uniqueness of fixed circles. Also, we provided some examples of self-mappings with fixed circles. On the other hand, we proved new fixed-circle results and applied the obtained results to the discontinuity problem and discontinuous activation functions [10].

Motivated by the above studies, our aim in this paper is to obtain some fixedcircle theorems for self-mappings on $S$-metric spaces. In Section 2., we recall some necessary definitions, lemmas and basic facts. In Section 3., we introduce the notion of a fixed circle on an $S$-metric space and then obtain some existence and uniqueness theorems for self-mappings having fixed circles via different techniques. We investigate the case in which the number of fixed circles is infinitely many. Some examples of self-mappings with fixed circles are given with a geometric viewpoint. Using Mathematica (Wolfram Research, Inc., Mathematica, Trial Version, Champaign, IL (2016)), we draw some figures related to the given examples.

\section{Preliminaries}

Definition 2.1. [12] Let $X$ be a nonempty set and $\mathcal{S}: X^{3} \rightarrow[0, \infty)$ be a function satisfying the following conditions for all $x, y, z, a \in X$.

1. $\mathcal{S}(x, y, z)=0$ if and only if $x=y=z$,

2. $\mathcal{S}(x, y, z) \leq \mathcal{S}(x, x, a)+\mathcal{S}(y, y, a)+\mathcal{S}(z, z, a)$. 
Then $S$ is called an $S$-metric on $X$ and the pair $(X, \mathcal{S})$ is called an $S$-metric space.

The following lemma can be considered as the symmetry condition and it will be used in the proofs of some theorems.

Lemma 2.1. [12] Let $(X, \mathcal{S})$ be an $S$-metric space. Then we have

$$
\mathcal{S}(x, x, y)=\mathcal{S}(y, y, x) .
$$

The relationships between a metric and an $S$-metric was given in what follows.

Lemma 2.2. [4] Let $(X, d)$ be a metric space. Then the following properties are satisfied:

1. $\mathcal{S}_{d}(x, y, z)=d(x, z)+d(y, z)$ for all $x, y, z \in X$ is an $S$-metric on $X$.

2. $x_{n} \rightarrow x$ in $(X, d)$ if and only if $x_{n} \rightarrow x$ in $\left(X, \mathcal{S}_{d}\right)$.

3. $\left\{x_{n}\right\}$ is Cauchy in $(X, d)$ if and only if $\left\{x_{n}\right\}$ is Cauchy in $\left(X, \mathcal{S}_{d}\right)$.

4. $(X, d)$ is complete if and only if $\left(X, \mathcal{S}_{d}\right)$ is complete.

The metric $\mathcal{S}_{d}$ was called an $S$-metric generated by $d[7]$. We know some examples of an $S$-metric which are not generated by any metric (see $[4,7,14]$ for more details).

On the other hand, Gupta claimed that every $S$-metric on $X$ defines a metric $d_{S}$ on $X$ as follows:

$$
d_{S}(x, y)=S(x, x, y)+S(y, y, x),
$$

for all $x, y \in X$ [3]. However, the function $d_{S}(x, y)$ defined in (2.1) does not always define a metric because the triangle inequality is not satisfied for all elements of $X$ everywhere (see [7] for more details).

The notions of an open ball, a closed ball and diameter were introduced on $S$-metric spaces as the following definitions.

Definition 2.2. [12] Let $(X, \mathcal{S})$ be an $S$-metric space. The open ball $B_{S}\left(x_{0}, r\right)$ and closed ball $B_{S}\left[x_{0}, r\right]$ with a center $x_{0}$ and a radius $r$ are defined by

$$
B_{S}\left(x_{0}, r\right)=\left\{x \in X: \mathcal{S}\left(x, x, x_{0}\right)<r\right\}
$$

and

$$
B_{S}\left[x_{0}, r\right]=\left\{x \in X: \mathcal{S}\left(x, x, x_{0}\right) \leq r\right\},
$$

for $r>0$ and $x_{0} \in X$. 
Definition 2.3. [6] Let $(X, \mathcal{S})$ be an $S$-metric space and $A$ be a nonempty subset of $X$. The diameter of $A$ is defined by

$$
\operatorname{diam}\{A\}=\sup \{\mathcal{S}(x, x, y): x, y \in A\} .
$$

If $A$ is $S$-bounded, then we will write $\operatorname{diam}\{A\}<\infty$.

Now we define the notion of a circle on an $S$-metric space.

Definition 2.4. Let $(X, \mathcal{S})$ be an $S$-metric space and $x_{0} \in X, r \in(0, \infty)$. We define the circle centered at $x_{0}$ with the radius $r$ as

$$
C_{x_{0}, r}^{S}=\left\{x \in X: \mathcal{S}\left(x, x, x_{0}\right)=r\right\}
$$

\section{Some Fixed-Circle Theorems on $S$-Metric Spaces}

In this section, we introduce the notion of a fixed circle on an $S$-metric space. Then we investigate some existence and uniqueness theorems for self-mappings having fixed circles.

Definition 3.1. Let $(X, \mathcal{S})$ be an $S$-metric space, $C_{x_{0}, r}^{S}$ be a circle on $X$ and $T: X \rightarrow X$ be a self-mapping. If $T x=x$ for all $x \in C_{x_{0}, r}^{S}$ then the circle $C_{x_{0}, r}^{S}$ is said to be a fixed circle of $T$.

\subsection{The existence of fixed circles}

We obtain some existence theorems for fixed circles of self-mappings.

Theorem 3.1. Let $(X, \mathcal{S})$ be an $S$-metric space and $C_{x_{0}, r}^{S}$ be any circle on $X$. Let us define the mapping

$$
\varphi: X \rightarrow[0, \infty), \varphi(x)=\mathcal{S}\left(x, x, x_{0}\right)
$$

for all $x \in X$. If there exists a self-mapping $T: X \rightarrow X$ satisfying

$$
\mathcal{S}(x, x, T x) \leq \varphi(x)+\varphi(T x)-2 r
$$

and

$$
\mathcal{S}(x, x, T x)+\mathcal{S}\left(T x, T x, x_{0}\right) \leq r,
$$

for all $x \in C_{x_{0}, r}^{S}$, then $C_{x_{0}, r}^{S}$ is a fixed circle of $T$. 
Proof. Let $x \in C_{x_{0}, r}^{S}$. Then using the conditions (3.2), (3.3), Lemma 2.1 and the triangle inequality, we get

$$
\begin{aligned}
\mathcal{S}(x, x, T x) & \leq \varphi(x)+\varphi(T x)-2 r \\
& =\mathcal{S}\left(x, x, x_{0}\right)+\mathcal{S}\left(T x, T x, x_{0}\right)-2 r \\
& \leq \mathcal{S}(x, x, T x)+\mathcal{S}(x, x, T x)+\mathcal{S}\left(T x, T x, x_{0}\right)+\mathcal{S}\left(T x, T x, x_{0}\right)-2 r \\
& =2 \mathcal{S}(x, x, T x)+2 \mathcal{S}\left(T x, T x, x_{0}\right)-2 r \\
& \leq 2 r-2 r=0
\end{aligned}
$$

and so

$$
\mathcal{S}(x, x, T x)=0,
$$

which implies $T x=x$. Consequently, $C_{x_{0}, r}^{S}$ is a fixed circle of $T$.

Remark 3.1. 1) Notice that the condition (3.2) guarantees that $T x$ is not in the interior of the circle $C_{x_{0}, r}^{S}$ for $x \in C_{x_{0}, r}^{S}$. Similarly, the condition (3.3) guarantees that $T x$ is not the exterior of the circle $C_{x_{0}, r}^{S}$ for $x \in C_{x_{0}, r}^{S}$. Hence $T x \in C_{x_{0}, r}^{S}$ for each $x \in C_{x_{0}, r}^{S}$ and so we get $T\left(C_{x_{0}, r}^{S}\right) \subset C_{x_{0}, r}^{S}$.

2) If an $S$-metric is generated by any metric $d$, then Theorem 3.1 can be used on the corresponding metric space.

3) The converse statement of Theorem 3.1 is also true.

Now we give an example of a self-mapping with a fixed circle.

Example 3.1. Let $X=\mathbb{R}$ and the function $\mathcal{S}: X^{3} \rightarrow[0, \infty)$ be defined by

$$
\mathcal{S}(x, y, z)=|x-z|+|y-z|,
$$

for all $x, y, z \in \mathbb{R}[13]$. Then $(X, \mathcal{S})$ is called the usual $S$-metric space. This $S$-metric is generated by the usual metric on $\mathbb{R}$. Let us consider the circle $C_{0,2}^{S}$ and define the self-mapping $T_{1}: \mathbb{R} \rightarrow \mathbb{R}$ as

$$
T_{1} x=\left\{\begin{array}{ccc}
x & \text { if } & x \in\{-1,1\} \\
10 & \text { otherwise }
\end{array},\right.
$$

for all $x \in \mathbb{R}$. Then the self-mapping $T_{1}$ satisfies the conditions (3.2) and (3.3). Hence $C_{0,2}^{S}=\{-1,1\}$ is a fixed circle of $T_{1}$.

Notice that $C_{\frac{9}{2}}^{S}, 11=\{-1,10\}$ is another fixed circle of $T_{1}$ and so the fixed circle is not unique for a giving self-mapping.

On the other hand, if we consider the usual metric $d$ on $\mathbb{R}$ then we obtain $C_{0,2}=$ $\{-2,2\}$. The circle $C_{0,2}$ is not a fixed circle of $T_{1}$.

Example 3.2. Let $X=\mathbb{R}^{2}$ and let the function $\mathcal{S}: X^{3} \rightarrow[0, \infty)$ be defined by

$$
\mathcal{S}(x, y, z)=\sum_{i=1}^{2}\left(\left|x_{i}-z_{i}\right|+\left|x_{i}+z_{i}-2 y_{i}\right|\right),
$$


for all $x=\left(x_{1}, x_{2}\right), y=\left(y_{1}, y_{2}\right)$ and $z=\left(z_{1}, z_{2}\right)$. Then it can be easily seen that $\mathcal{S}$ is an $S$-metric on $\mathbb{R}^{2}$, which is not generated by any metric, and the pair $\left(\mathbb{R}^{2}, \mathcal{S}\right)$ is an $S$-metric space.

Let us consider the unit circle $C_{0,1}^{S}$ and define the self-mapping $T_{2}: \mathbb{R} \rightarrow \mathbb{R}$ as

$$
T_{2} x=\left\{\begin{array}{c}
x \\
(1,0)
\end{array} \quad \text { if } \quad \begin{array}{c}
x \in C_{0,1}^{S} \\
\text { otherwise }
\end{array},\right.
$$

for all $x \in \mathbb{R}^{2}$. Then the self-mapping $T_{2}$ satisfies the conditions (3.2) and (3.3). Therefore $C_{0,1}^{S}$ is a fixed circle of $T_{2}$ as shown in Figure 3.1.

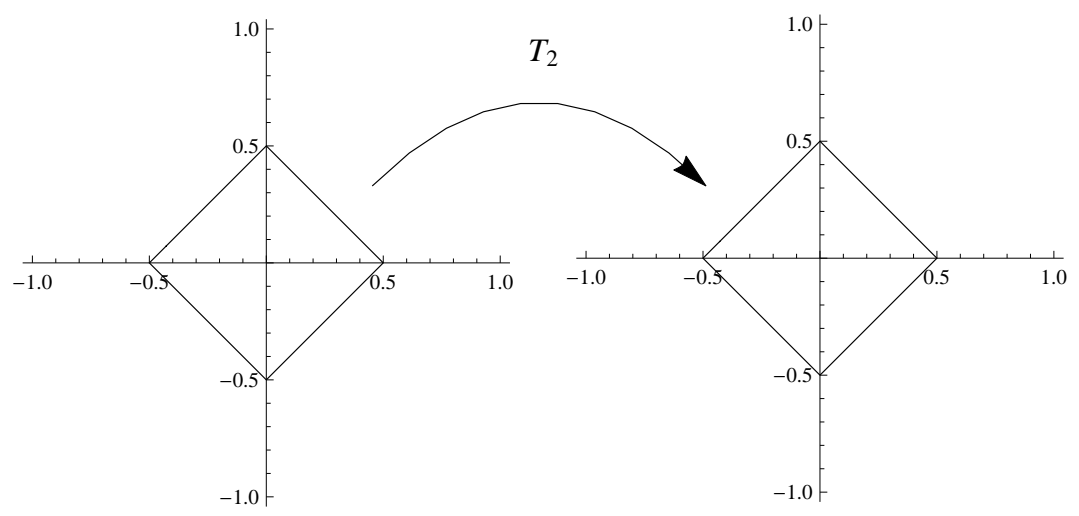

Fig. 3.1: The fixed circle of $T_{2}$.

In the following example, we give an example of a self-mapping which satisfies the condition (3.2) and does not satisfy the condition (3.3).

Example 3.3. Let $X=\mathbb{R}$ and the function $\mathcal{S}: X^{3} \rightarrow[0, \infty)$ be defined by

$$
\mathcal{S}(x, y, z)=|x-z|+|x+z-2 y|,
$$

for all $x, y, z \in \mathbb{R}[7]$. Then $\mathcal{S}$ is an $S$-metric which is not generated by any metric and $(X, \mathcal{S})$ is an $S$-metric space. Let us consider the circle $C_{0,3}^{S}$ and define the self-mapping $T_{3}: \mathbb{R} \rightarrow \mathbb{R}$ as

$$
T_{3} x=\left\{\begin{array}{ccc}
-\frac{7}{2} & \text { if } & x=-\frac{3}{2} \\
\frac{7}{2} & \text { if } & x=\frac{3}{2} \\
7 & & \text { otherwise }
\end{array},\right.
$$


for all $x \in \mathbb{R}$. Then the self-mapping $T_{3}$ satisfies the condition (3.2) but does not satisfy the condition (3.3). Clearly $T_{3}$ does not fix the circle $C_{0,3}^{S}$.

In the following example, we give an example of a self-mapping which satisfies the condition (3.3) and does not satisfy the condition (3.2).

Example 3.4. Let $(X, \mathcal{S})$ be an $S$-metric space, $C_{x_{0}, r}^{S}$ be a circle on $X$ and the selfmapping $T_{4}: X \rightarrow X$ be defined as

$$
T_{4} x=x_{0},
$$

for all $x \in X$. Then the self-mapping $T_{4}$ satisfies the condition (3.3) but does not satisfy the condition (3.2). Clearly $T_{4}$ does not fix the circle $C_{x_{0}, r}^{S}$.

Now we give another existence theorem for fixed circles.

Theorem 3.2. Let $(X, \mathcal{S})$ be an $S$-metric space and $C_{x_{0}, r}^{S}$ be any circle on $X$. Let the mapping $\varphi$ be defined as (3.1). If there exists a self-mapping $T: X \rightarrow X$ satisfying

and

$$
\mathcal{S}(x, x, T x) \leq \varphi(x)-\varphi(T x)
$$

for all $x \in C_{x_{0}, r}^{S}$ and some $h \in[0,1)$, then $C_{x_{0}, r}^{S}$ is a fixed circle of $T$.

Proof. Let $x \in C_{x_{0}, r}^{S}$. On the contrary, assume that $x \neq T x$. Then using the conditions (3.4) and (3.5), we obtain

$$
\begin{aligned}
\mathcal{S}(x, x, T x) & \leq \varphi(x)-\varphi(T x) \\
& =\mathcal{S}\left(x, x, x_{0}\right)-\mathcal{S}\left(T x, T x, x_{0}\right) \\
& =r-\mathcal{S}\left(T x, T x, x_{0}\right) \\
& \leq h \mathcal{S}(x, x, T x)+\mathcal{S}\left(T x, T x, x_{0}\right)-\mathcal{S}\left(T x, T x, x_{0}\right) \\
& =h \mathcal{S}(x, x, T x),
\end{aligned}
$$

which is a contradiction since $h \in[0,1)$. Hence we get $T x=x$ and $C_{x_{0}, r}^{S}$ is a fixed circle of $T$.

Remark 3.2. 1) Notice that the condition (3.4) guarantees that $T x$ is not in the exterior of the circle $C_{x_{0}, r}^{S}$ for $x \in C_{x_{0}, r}^{S}$. Similarly, the condition (3.5) shows that $T x$ can lie on either the exterior or the interior of the circle $C_{x_{0}, r}^{S}$ for $x \in C_{x_{0}, r}^{S}$. Hence $T x$ should lie on the interior of the circle $C_{x_{0}, r}^{S}$.

2 ) If an $S$-metric is generated by any metric $d$, then Theorem 3.2 can be used on the corresponding metric space.

3) The converse statement of Theorem 3.2 is also true.

Now we give some examples of self-mappings which have a fixed-circle. 


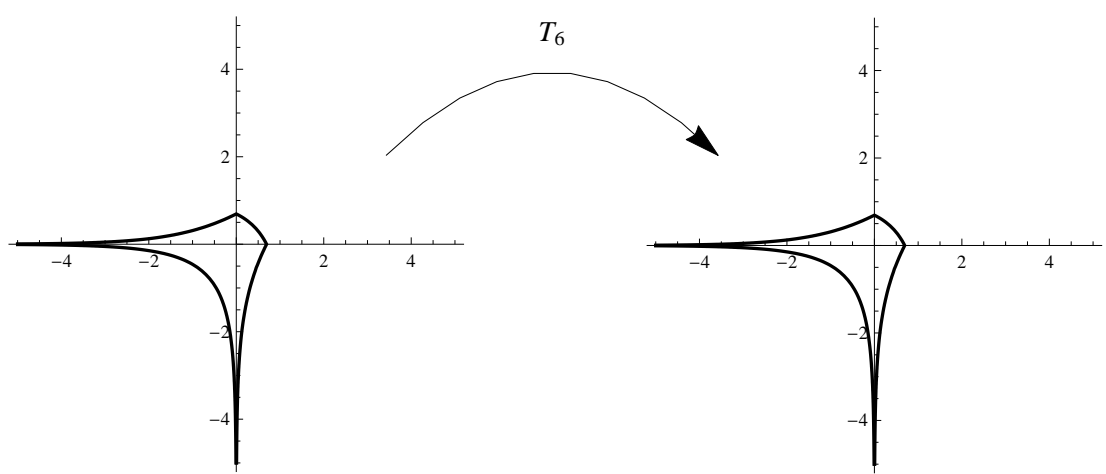

FIG. 3.2: The fixed circle of $T_{6}$.

Example 3.5. Let $X=\mathbb{R}$ and $(X, \mathcal{S})$ be the usual $S$-metric space. Let us consider the circle $C_{1,2}^{S}=\{0,2\}$ and define the self-mapping $T_{5}: \mathbb{R} \rightarrow \mathbb{R}$ as

$$
T_{5} x=\left\{\begin{array}{ccc}
e^{x}-1 & \text { if } & x=0 \\
2 x-2 & \text { if } & x=2 \\
3 & & \text { otherwise }
\end{array},\right.
$$

for all $x \in \mathbb{R}$. Then the self-mapping $T_{5}$ satisfies the conditions (3.4) and (3.5). Hence $C_{1,2}^{S}$ is a fixed circle of $T_{5}$.

On the other hand, if we consider the usual metric $d$ on $\mathbb{R}$ then we have $C_{1,2}=\{-1,3\}$. The circle $C_{1,2}$ is not a fixed circle of $T_{5}$. But $C_{1,1}=\{0,2\}$ is a fixed circle of $T_{5}$ on $(X, d)$.

Example 3.6. Let $X=\mathbb{R}^{2}$ and let the function $\mathcal{S}: X^{3} \rightarrow[0, \infty)$ be defined by

$$
\mathcal{S}(x, y, z)=\sum_{i=1}^{2}\left(\left|e^{x_{i}}-e^{z_{i}}\right|+\left|e^{x_{i}}+e^{z_{i}}-2 e^{y_{i}}\right|\right),
$$

for all $x=\left(x_{1}, x_{2}\right), y=\left(y_{1}, y_{2}\right)$ and $z=\left(z_{1}, z_{2}\right)$. Then it can be easily checked that $\mathcal{S}$ is an $S$-metric on $\mathbb{R}^{2}$, which is not generated by any metric, and the pair $\left(\mathbb{R}^{2}, \mathcal{S}\right)$ is an $S$-metric space.

Let us consider the circle $C_{x_{0}, r}^{S}$ centered at $x_{0}=(0,0)$ with the radius $r=2$ and define the self-mapping $T_{6}: \mathbb{R} \rightarrow \mathbb{R}$ as

$$
T_{6} x=\left\{\begin{array}{c}
x \\
(\ln 2,0)
\end{array} \quad \text { if } \quad \begin{array}{c}
x \in C_{0,2}^{S} \\
\text { otherwise }
\end{array},\right.
$$


for all $x \in \mathbb{R}^{2}$. Then the self-mapping $T_{6}$ satisfies the conditions (3.4) and (3.5). Therefore $C_{0,2}^{S}$ is the fixed circle of $T_{6}$ as shown in Figure 3.2.

In the following example, we give an example of a self-mapping which satisfies the condition (3.4) and does not satisfy the condition (3.5).

Example 3.7. Let $(X, \mathcal{S})$ be an $S$-metric space and $C_{x_{0}, r}^{S}$ be a circle on $X$. If we consider the self-mapping $T_{4} x=x_{0}$, then the self-mapping $T_{4}$ satisfies the condition (3.4) but does not satisfy the condition (3.5). It can be easily seen that $T_{4}$ does not fix a circle $C_{x_{0}, r}^{S}$.

In the following example, we give an example of a self-mapping which satisfies the condition (3.5) and does not satisfy the condition (3.4).

Example 3.8. Let $X=\mathbb{R}$ and $(X, \mathcal{S})$ be an $S$-metric space with an $S$-metric defined as in Example 3.3. Let us consider the unit circle $C_{0,1}^{S}$ and define the self-mapping $T_{7}: \mathbb{R} \rightarrow \mathbb{R}$ as

$$
T_{7} x=1
$$

for all $x \in \mathbb{R}$. Then the self-mapping $T_{7}$ satisfies the condition (3.5) but does not satisfy the condition (3.4). It can be easily shown that $T_{7}$ does not fix the unit circle $C_{0,1}^{S}$.

Let $I_{X}: X \rightarrow X$ be the identity map defined as $I_{X}(x)=x$ for all $x \in X$. Notice that the identity map satisfies the conditions (3.2) and (3.3) (resp. (3.4) and (3.5)) in Theorem 3.1 (resp. Theorem 3.2) for any circle. Now we determine a condition which excludes the $I_{X}$ from Theorem 3.1 and Theorem 3.2. For this purpose, we give the following theorem.

Theorem 3.3. Let $(X, \mathcal{S})$ be an $S$-metric space, $T: X \rightarrow X$ be a self mapping having a fixed circle $C_{x_{0}, r}^{S}$ and the mapping $\varphi$ be defined as (3.1). The self-mapping $T$ satisfies the condition

$$
\left(I_{S}\right) \quad \mathcal{S}(x, x, T x) \leq \frac{\varphi(x)-\varphi(T x)}{h},
$$

for all $x \in X$ and some $h>2$ if and only if $T=I_{X}$.

Proof. Let $x \in X$ be an arbitrary element. Then using the inequality $\left(I_{S}\right)$, Lemma 2.1 and triangle inequality, we obtain

$$
\begin{aligned}
h \mathcal{S}(x, x, T x) & \leq \varphi(x)-\varphi(T x) \\
& =\mathcal{S}\left(x, x, x_{0}\right)-\mathcal{S}\left(T x, T x, x_{0}\right) \\
& \leq 2 \mathcal{S}(x, x, T x)+\mathcal{S}\left(T x, T x, x_{0}\right)-\mathcal{S}\left(T x, T x, x_{0}\right) \\
& =2 \mathcal{S}(x, x, T x)
\end{aligned}
$$

and so

$$
(h-2) \mathcal{S}(x, x, T x) \leq 0 .
$$


Since $h>2$ it should be $\mathcal{S}(x, x, T x)=0$ and so $T x=x$. Consequently, we obtain $T=I_{X}$

Conversely, it is clear that the identity map $I_{X}$ satisfies the condition $\left(I_{S}\right)$.

Remark 3.3. 1) If a self-mapping $T$, which has a fixed circle, satisfies the conditions (3.2) and (3.3) (resp. (3.4) and (3.5)) in Theorem 3.1 (resp. Theorem 3.2) but does not satisfy the condition $\left(I_{S}\right)$ in Theorem 3.3 then the self-mapping $T$ cannot be an identity map.

2) If an $S$-metric is generated by any metric $d$, then Theorem 3.3 can be used on the corresponding metric space.

\subsection{The uniqueness of fixed circles}

We investigate the uniqueness conditions of fixed circles given in the existence theorems. For any given circles $C_{x_{0}, r}^{S}$ and $C_{x_{1}, \rho}^{S}$ on $X$, we notice that there exists at least one self-mapping $T$ of $X$ such that $T$ fixes the circles $C_{x_{0}, r}^{S}, C_{x_{1}, \rho}^{S}$. Indeed let us define the mappings $\varphi_{1}, \varphi_{2}: X \rightarrow[0, \infty)$ as

$$
\varphi_{1}(x)=\mathcal{S}\left(x, x, x_{0}\right)
$$

and

$$
\varphi_{2}(x)=\mathcal{S}\left(x, x, x_{1}\right),
$$

for all $x \in X$. If we define the self-mapping $T_{8}: X \rightarrow X$ as

$$
T_{8} x=\left\{\begin{array}{ccc}
x & \text { if } & x \in C_{x_{0}, r}^{S} \cup C_{x_{1}, \rho}^{S} \\
\alpha & & \text { otherwise }
\end{array},\right.
$$

for all $x \in X$, where $\alpha$ is a constant satisfying $S\left(\alpha, \alpha, x_{0}\right) \neq r$ and $S\left(\alpha, \alpha, x_{1}\right) \neq \rho$, it can be easily seen that the self-mapping $T_{8}: X \rightarrow X$ satisfies the conditions (3.2) and (3.3) in Theorem 3.1 (resp. (3.4) and (3.5) in Theorem 3.2) for the circles $C_{x_{0}, r}^{S}$ and $C_{x_{1}, \rho}^{S}$ using the mappings $\varphi_{1}$ and $\varphi_{2}$, respectively. Hence $T_{8}$ fixes both of the circles $C_{x_{0}, r}^{S}$ and $C_{x_{1}, \rho}^{S}$. In this way, the number of fixed circles can be extended to any positive integer $n$ using the same arguments.

In the following example, the self-mapping $T_{9}$ has two fixed circle.

Example 3.9. Let $X=\mathbb{R}$ and $(X, \mathcal{S})$ be an $S$-metric space with the $S$-metric defined in Example 3.3. Let us consider the circles $C_{0,2}^{S}, C_{0,4}^{S}$ and define the self-mapping $T_{9}: \mathbb{R} \rightarrow \mathbb{R}$ as

$$
T_{9} x=\left\{\begin{array}{ccc}
x & \text { if } & x \in\{-2,-1,1,2\} \\
\alpha & & \text { otherwise }
\end{array},\right.
$$

for all $x \in X$ where $\alpha \in X$. Then the conditions (3.2) and (3.3) are satisfied by $T_{9}$ for the circles $C_{0,2}^{S}$ and $C_{0,4}^{S}$, respectively. Consequently, $C_{0,2}^{S}$ and $C_{0,4}^{S}$ are the fixed circles of $T_{9}$. 
Now we investigate the uniqueness conditions for the fixed circles in Theorem 3.1 using Rhoades' contractive condition on $S$-metric spaces.

Theorem 3.4. Let $(X, \mathcal{S})$ be an $S$-metric space and $C_{x_{0}, r}^{S}$ be any circle on $X$. Let $T: X \rightarrow X$ be a self-mapping satisfying the conditions (3.2) and (3.3) given in Theorem 3.1. If the contractive condition

$$
\begin{gathered}
\mathcal{S}(T x, T x, T y)<\max \{\mathcal{S}(x, x, y), \mathcal{S}(T x, T x, x), \mathcal{S}(T y, T y, y), \\
\mathcal{S}(T y, T y, x), \mathcal{S}(T x, T x, y)\}
\end{gathered}
$$

is satisfied for all $x \in C_{x_{0}, r}^{S}, y \in X \backslash C_{x_{0}, r}^{S}$ by $T$, then $C_{x_{0}, r}^{S}$ is a unique fixed circle of $T$.

Proof. Suppose that there exist two fixed circles $C_{x_{0}, r}^{S}$ and $C_{x_{1}, \rho}^{S}$ of the self-mapping $T$, that is, $T$ satisfies the conditions (3.2) and (3.3) for each circles $C_{x_{0}, r}^{S}$ and $C_{x_{1}, \rho}^{S}$. Let $x \in C_{x_{0}, r}^{S}$ and $y \in C_{x_{1}, \rho}^{S}$ be arbitrary points with $x \neq y$. Using the contractive condition (3.6), we obtain

$$
\begin{aligned}
\mathcal{S}(x, x, y)= & \mathcal{S}(T x, T x, T y)<\max \{\mathcal{S}(x, x, y), \mathcal{S}(T x, T x, x), \mathcal{S}(T y, T y, y), \\
& \mathcal{S}(T y, T y, x), \mathcal{S}(T x, T x, y)\} \\
= & \mathcal{S}(x, x, y)
\end{aligned}
$$

which is a contradiction. Hence it should be $x=y$. Consequently, $C_{x_{0}, r}^{S}$ is the unique fixed circle of $T$.

The following example shows that the circle $C_{x_{0}, r}^{S}$ is not necessarily unique in Theorem 3.2.

Example 3.10. Let $(X, \mathcal{S})$ be an $S$-metric space and $C_{x_{1}, r_{1}}, \cdots, C_{x_{n}, r_{n}}$ be any circles on $X$. Let us define the self-mapping $T_{10}: X \rightarrow X$ as

$$
T_{10} x=\left\{\begin{array}{ccc}
x & \text { if } & x \in \bigcup_{i=1}^{n} C_{x_{i}, r_{i}} \\
x_{0} & & \text { otherwise }
\end{array},\right.
$$

for all $x \in X$, where $x_{0}$ is a constant in $X$. Then it can be easily checked that the conditions (3.4) and (3.5) are satisfied by $T_{10}$ for the circles $C_{x_{1}, r_{1}}, \cdots, C_{x_{n}, r_{n}}$, respectively. Consequently, the circles $C_{x_{1}, r_{1}}, \cdots, C_{x_{n}, r_{n}}$ are fixed circles of $T_{10}$. Notice that these circles do not have to be disjoint.

Now we give the following uniqueness theorem for the fixed circles in Theorem 3.2 using the notion of diameter on $S$-metric spaces.

Theorem 3.5. Let $(X, \mathcal{S})$ be an $S$-metric space, $C_{x_{0}, r}^{S}$ be any circle on $X, U_{x}=$ $\left\{T^{n} x: n \in \mathbb{N}\right\}, U_{y}=\left\{T^{n} y: n \in \mathbb{N}\right\}$, $\operatorname{diam}\left\{U_{x}\right\}<\infty$ and $\operatorname{diam}\left\{U_{y}\right\}<\infty$. Let 
$T: X \rightarrow X$ be a self-mapping satisfying the conditions (3.4) and (3.5) given in Theorem 3.2. If the contractive condition

$$
\mathcal{S}(T x, T x, T y)<\operatorname{diam}\left\{U_{x} \cup U_{y}\right\},
$$

is satisfied for all $x \in C_{x_{0}, r}^{S}, y \in X \backslash C_{x_{0}, r}^{S}$ by $T$, then $C_{x_{0}, r}^{S}$ is the unique fixed circle of $T$.

Proof. Assume that there exist two fixed circles $C_{x_{0}, r}^{S}$ and $C_{x_{1}, \rho}^{S}$ of the self-mapping $T$, that is, $T$ satisfies the conditions (3.4) and (3.5) for each circles $C_{x_{0}, r}^{S}$ and $C_{x_{1}, \rho}^{S}$. Let $x \in C_{x_{0}, r}^{S}$ and $y \in C_{x_{1}, \rho}^{S}$ be arbitrary points with $x \neq y$. Using the contractive condition (3.7), we obtain

$$
\mathcal{S}(x, x, y)=\mathcal{S}(T x, T x, T y)<\operatorname{diam}\left\{U_{x} \cup U_{y}\right\}=\mathcal{S}(x, x, y),
$$

which is a contradiction. Hence it should be $x=y$. Consequently, $C_{x_{0}, r}^{S}$ is the unique fixed circle of $T$.

\subsection{Infinity of fixed circles}

We give a new approach to obtain fixed-circle results. To do this, let us denote by $R_{S}(x, y)$ the right side of the inequality $(S 25)$. Using the number $R_{S}(x, y)$, we obtain the following theorem. This theorem generates many (finite or infinite) fixed circles for a given self-mapping.

Theorem 3.6. Let $(X, \mathcal{S})$ be an $S$-metric space, $T: X \rightarrow X$ be a self-mapping and $r=\inf \{\mathcal{S}(T x, T x, x): T x \neq x\}$. If there exists a point $x_{0} \in X$ satisfying

$$
\mathcal{S}(x, x, T x)<R_{S}\left(x, x_{0}\right)
$$

for all $x \in X$ when $\mathcal{S}(T x, T x, x)>0$ and

$$
\mathcal{S}\left(T x, T x, x_{0}\right)=r
$$

for all $x \in C_{x_{0}, r}^{S}$, then $C_{x_{0}, r}^{S}$ is a fixed circle of $T$. The self-mapping $T$ also fixes the closed ball $B_{S}\left[x_{0}, r\right]$.

Proof. Let $x \in C_{x_{0}, r}^{S}$ and $T x \neq x$. Then using the inequality (3.8) and Lemma 2.1, we get

$$
=\max \left\{\begin{array}{c}
\mathcal{S}(x, x, T x)<R_{S}\left(x, x_{0}\right) \\
\mathcal{S}\left(x, x, x_{0}\right), \mathcal{S}(T x, T x, x), \mathcal{S}\left(T x_{0}, T x_{0}, x_{0}\right), \\
\mathcal{S}\left(T x_{0}, T x_{0}, x\right), \mathcal{S}\left(T x, T x, x_{0}\right)
\end{array}\right\} .
$$

At first, using the inequality (3.10) and Lemma 2.1, we show $T x_{0}=x_{0}$. Suppose that $T x_{0} \neq x_{0}$. For $x=x_{0}$, we obtain

$$
\begin{aligned}
\mathcal{S}\left(x_{0}, x_{0}, T x_{0}\right) & <R_{S}\left(x_{0}, x_{0}\right) \\
& =\max \left\{\begin{array}{c}
\mathcal{S}\left(x_{0}, x_{0}, x_{0}\right), \mathcal{S}\left(T x_{0}, T x_{0}, x_{0}\right), \mathcal{S}\left(T x_{0}, T x_{0}, x_{0}\right), \\
\mathcal{S}\left(T x_{0}, T x_{0}, x_{0}\right), \mathcal{S}\left(T x_{0}, T x_{0}, x_{0}\right)
\end{array}\right\} \\
& =\mathcal{S}\left(T x_{0}, T x_{0}, x_{0}\right)=\mathcal{S}\left(x_{0}, x_{0}, T x_{0}\right),
\end{aligned}
$$


a contradiction. It should be $T x_{0}=x_{0}$. Then by the inequality (3.10), the condition (3.9), definition of $r$ and Lemma 2.1, we have

$$
\begin{aligned}
\mathcal{S}(x, x, T x) & <\max \left\{\begin{array}{c}
\mathcal{S}\left(x, x, x_{0}\right), \mathcal{S}(T x, T x, x), \mathcal{S}\left(x_{0}, x_{0}, x_{0}\right), \\
\mathcal{S}\left(x_{0}, x_{0}, x\right), \mathcal{S}\left(T x, T x, x_{0}\right)
\end{array}\right\} \\
& =\max \{r, \mathcal{S}(T x, T x, x)\}=\mathcal{S}(T x, T x, x)=\mathcal{S}(x, x, T x),
\end{aligned}
$$

a contradiction. Therefore we get $T x=x$, that is, $C_{x_{0}, r}^{S}$ is a fixed circle of $T$.

Finally we prove that $T$ fixes the closed ball $B_{S}\left[x_{0}, r\right]$. To do this, we show that $T$ fixes any circle $C_{x_{0}, \rho}^{S}$ with $\rho<r$. Let $x \in C_{x_{0}, \rho}^{S}$ and $T x \neq x$. From the similar arguments used in the above, we have $T x=x$.

We give the following example.

Example 3.11. Let $X=\mathbb{R}$ be the usual $S$-metric space. Let us define the self-mapping $T: \mathbb{R} \rightarrow \mathbb{R}$ as

$$
T x=\left\{\begin{array}{ccc}
x & \text { if } & |x|<3 \\
x+2 & \text { if } & |x| \geq 3
\end{array},\right.
$$

for all $x \in \mathbb{R}$. The self-mapping $T$ satisfies the conditions of Theorem 3.6 with $x_{0}=0$. Indeed, we get

$$
\mathcal{S}(x, x, T x)=2|x-T x|=4>0,
$$

for all $x \in \mathbb{R}$ such that $|x| \geq 3$. Then we have

$$
\begin{aligned}
R_{S}(x, 0) & =\max \{\mathcal{S}(x, x, 0), \mathcal{S}(T x, T x, x), \mathcal{S}(0,0,0), \mathcal{S}(0,0, x), \mathcal{S}(T x, T x, 0)\} \\
& =\max \{2|x|, 4,0,2|x|, 2|x+2|\} \\
& =\max \{2|x|, 2|x+2|\}
\end{aligned}
$$

and so

$$
\mathcal{S}(x, x, T x)<R_{S}(x, 0)
$$

Therefore the condition (3.8) is satisfied. We also obtain

$$
r=\min \{\mathcal{S}(T x, T x, x): T x \neq x\}=4 .
$$

It can be easily seen that the condition (3.9) is satisfied by $T$. Consequently, $T$ fixes the circle $C_{0,4}^{S}=\{x \in \mathbb{R}:|x|=2\}$ and the closed ball $B_{S}[0,4]=\{x \in \mathbb{R}:|x| \leq 2\}$.

Remark 3.4. 1) Notice that the condition (3.9) guarantees that $T x \in C_{x_{0}, r}^{S}$ for each $x \in C_{x_{0}, r}^{S}$ and so $T\left(C_{x_{0}, r}^{S}\right) \subset C_{x_{0}, r}^{S}$.

2) The self-mapping $T$ defined in Example 3.11 has other fixed circles. Theorem 3.6 gives us some of these circles.

3) A self-mapping $T$ can fix infinitely many circles (see Example 3.11).

The converse statement is not always true as seen in the following example.

Example 3.12. Let $x_{0} \in X$ be any point. If we define the self-mapping $T: X \rightarrow X$ as

$$
T x=\left\{\begin{array}{ccc}
x & \text { if } & x \in B_{S}\left[x_{0}, \mu\right] \\
x_{0} & \text { if } & x \notin B_{S}\left[x_{0}, \mu\right]
\end{array},\right.
$$

for all $x \in X$ with $\mu>0$, then $T$ does not satisfies the condition (3.8), but $T$ fixes every circle $C_{x_{0}, \rho}^{S}$ with $\rho \leq \mu$. 


\section{R E F E R E N C E S}

1. S. BANACH: Sur les operations dans les ensembles abstraits et leur application aux equations integrals. Fund. Math. 2 (1922), 133-181.

2. K. Ciesielski: On Stefan Banach and some of his results. Banach J. Math. Anal. 1 (1) (2007), 1-10.

3. A. Gupta: Cyclic contraction on S-metric space. Int. J. Anal. Appl. 3 (2) (2013), 119-130.

4. N. T. Hieu, N. T. Ly and N. V. Dung: A Generalization of Ciric Quasi-Contractions for Maps on S-Metric Spaces. Thai J. Math. 13 (2) (2015), 369-380.

5. N. Özdemir, B. B. İskender and N. Y. ÖzGür: Complex valued neural network with Möbius activation function. Commun. Nonlinear Sci. Numer. Simul. 16 (12) (2011), $4698-4703$.

6. N. Y. ÖZGür and N. TAŞ: Some fixed point theorems on S-metric spaces. Mat. Vesnik 69 (1) (2017), 39-52.

7. N. Y. ÖzGür and N. TAŞ: Some new contractive mappings on S-metric spaces and their relationships with the mapping (S25). Math. Sci. 11 (1) (2017), 7-16.

8. N. Y. ÖzGür and N. TAŞ: Some generalizations of fixed point theorems on S-metric spaces. Essays in Mathematics and Its Applications in Honor of Vladimir Arnold, New York, Springer, 2016.

9. N. Y. ÖzGür and N. TAŞ: Some fixed-circle theorems on metric spaces. Bull. Malays. Math. Sci. Soc. (2017). https://doi.org/10.1007/s40840-017-0555-z

10. N. Y. ÖzGür and N. TAŞ: Some fixed-circle theorems and discontinuity at fixed circle. AIP Conference Proceedings 1926, 020048 (2018).

11. B. E. RhoAdes: A comparison of various definitions of contractive mappings. Trans. Amer. Math. Soc. 226 (1977), 257-290.

12. S. Sedghi, N. Shobe and A. Aliouche: A generalization of fixed point theorems in S-metric spaces: Mat. Vesnik 64 (3) (2012), 258-266.

13. S. Sedghi and N. V. Dung: Fixed point theorems on S-metric spaces. Mat. Vesnik 66 (1) (2014), 113-124.

14. N. TAŞ: Fixed point theorems and their various applications. $\mathrm{Ph}$. D. Thesis, University of Balıkesir, Balıkesir, 2017.

Nihal Y. Özgür

Faculty of Arts and Sciences

Department of Mathematics

10145 Balıkesir, Turkey

nihal@balikesir.edu.tr

Nihal Taş

Faculty of Arts and Sciences

Department of Mathematics

10145 Balıkesir, Turkey

nihaltas@balikesir.edu.tr 